\title{
Development of a New Heat Transfer Optimization Method for Compressible Fluid Flows and
}

\section{it Numerical Verifications}

\begin{abstract}
A general optimization criterion for heat transfer process is developed based on the entransy theory. Then, a modified field synergy principle for compressible viscous fluid, which represents the irreversibility of the specific heat transfer processes, is discussed. Modified field synergy equations are set up and theoretically, the solution of the modified field synergy equations under the constraint conditions of a given mean kinetic energy is able to show the optimal flow field, in which the amount of heat transfer is maximized. In the study, a numerical simulation of the heat transfer processes of air in a cavity is selected as the numerical verification of the modified field synergy equations. Numerical simulation results show that asymmetric vortices are generated in the flow field due to the variation of the density. These vortices enhance the heat transfer performance in the absence of too much work dissipation. Comparisons among the results of the Navier-Stokes equations, the existed laminar field synergy equations, and the modified field synergy equations are made. The results confirm that the equations developed in this study correspond to the best heat-transfer performance when the density of fluid changes with temperature or pressure.
\end{abstract}

Keywords: Compressible fluid, Variable property, Field synergy equation, Entransy dissipation, Optimization 


\section{Introduction}

More than $90 \%$ of all the worldwide energy utilization involves the heat transfer process. That is to say, there is a huge potential for conserving energy on thermal engineering through heat-transfer efficiency techniques. Scholars have been working for centuries seeking for the efficient ways to improve the heat transfer performance. During the last several decades, a large number of convective heat-transfer enhancement technologies have been developed including using extended surfaces, spoilers, stirrers, and external electric or magnetic field [1-3]. These trials have successfully reduced energy consumption and retrench expenditure of equipment, but the mechanism of heat transfer process is still unclear. Indeed, none of these analyses adequately explain the principle of heat transfer enhancement, and engineering heat transfer is still considered to be an experimental problem.

In the 1980s, Bejan $[4,5]$ introduced the entropy generation function for estimating the irreversibility of convective heat-transfer processes in a system along finite temperature gradient with viscous effects. Then convective heat-transfer processes were optimized with the objective of minimum entropy generation. The minimum entropy generation principle is well accepted and widely used in processes analysis and system optimization [6-8]. As time goes by, people found that the entropy generation could not always reach its minimum value when the system performs best, and this weird phenomenon was called "the entropy generation paradox" by Bejan himself. The entropy generation paradox shows that problems may occur during the usage of entropy generation to describe the irreversibility of the heat transfer process in heat exchangers [9-11]. A recent published research indicates that the entropy generation results as a maximum when it is evaluated by the exterior surroundings of the system and a minimum when it is evaluated within the system [12]. The system considered was a typical energy transduction system, that is to say, the paradox found in the heat exchanger system hasn’t been explained.

Another way to consider the possibility of heat transfer optimization is the definition of entransy [13, 14]. In this theory, entransy is defined as the heat transfer ability. It is found that in the irreversible processes entransy is dissipated 
and heat transfer capability attenuates [15]. This phenomena leads to the extreme entransy dissipation principle: the extreme entransy dissipation corresponds to the minimum transfer resistant for a given heat-transfer problem. The entransy dissipation of a heat transfer process can serve as a judgment of the performance of heat exchanger. Based on this theory, several types of heat-transfer processes, including heat conduction [14], convective heat transfer [15, 16] and thermal radiation [17], have been analyzed and optimized numerically. The scholars want to extend this theory into a much more extreme condition to show the general applicability of it, but according to the author's knowledge, the deductions and applications published are all under an incompressible assumption.

The variable-property of fluid may greatly improve the system performance if the system is carefully designed. A good example is the supercritical fluid. The densities of a supercritical fluid are subject to change when pressure or temperature is tampered with. This changing of properties makes supercritical fluid disparate from regular fluids, and thus become a favorite in heat transfer enhancement and thermodynamic cycles for efficiency improvement [18-20]. Thanks to the mature development of modern computation technology, e.g., the computational fluid dynamics and numerical heat transfer methods, robust treatment on the variable-property flow and heat transfer problems could be implemented. In the last decade, the effects of temperature-dependent fluid property were emphasized in many numerical researches, even in micro scale and pore-scale [21-24]. These simulations portrayed the flow details and showed the advantages of the variable-property.

From the aforementioned discussion, the concept of entransy has been developed and leads to a method for convective heat-transfer optimization. Chen et al. [25] investigate the physical essential and the applicability of the extreme entransy dissipation principle in convective heat-transfer optimization. And developed field synergy equation for laminar (LFSE) and turbulent (TFSE) [16] heat transfer processes. By solving the field synergy equation under a specific viscous dissipation value, the optimal flow field can be obtained. According to the theory, the optimal flow field indicates the flow pattern when the heat transfer rate is maximized, which gives a guidance to the further industrial application. 
However, all the discussions up to now are based on a constant volume assumption, which means the fluids considered in these researches are all incompressible. Researching results showed that the flow and heat transfer performance maybe very different from the regular ones because of the high compressibility [26, 27]. When the fluid is compressible, vortexes are generated even when the inlet Reynolds number is not very high, which may either enhance or subdued the heat transfer performance [28]. This phenomenon is interesting as well as disturbing, since we don't know which kind of vortexes can lead to a better heat transfer performance. The current field synergy equations can give an optimal flow field only under the incompressible assumption, so we want to make a complement of the current entransy discussion and extend to compressible fluid and variable fluid properties.

The current study is an extended analysis of the optimization method of a compressible heat transfer process. It's an attempt to develop the existed entransy theory and the laminar field synergy equations. The optimization criteria for the heat transfer process of compressible viscous fluid with variable physical properties have been stated. We introduce the modified field synergy equations by presenting reasonable derivations under the assumption of compressible flow and variable physical properties. By solving the modified field synergy equations, the flow pattern corresponding to the maximum heat transfer rate under a constant viscous dissipation can be obtained. Numerical verifications are done to optimize the heat transfer processes of air in cavity by using the CFD method. It's hoped the models and methods developed could bring a different view in the heat transfer optimization.

\section{Theoretical and Mathematical Models}

2.1 Optimization criteria for the heat transfer process of compressible viscous fluid

This part needs to be discussed first because the later derivation is based on the definition of the optimization criteria.

As it's said before, the existed discussions of entransy theory are all under the assumption of constant volume, and the 
criteria of extreme entransy dissipation were used to obtain the optimized flow field in the former works [16]. When it comes to the compressible fluid, the optimization criteria need to be modified to suit the striking changes of density. For compressible fluid, volume variations may be involved in a heat conduction or convection process. In order to simplify the discussion, material bodies are used in the later derivations to avoid the effect of mass into and out of the bodies.

The general energy conservation equation can be expressed as,

$$
\frac{d U}{d t}=-p \frac{d}{d t}\left(\frac{1}{\rho}\right)+\frac{1}{\rho} \phi_{m o m}+\frac{1}{\rho} \frac{d q^{e}}{d t}+\frac{1}{\rho} \dot{Q}
$$

where $U$ is the internal energy of the body, $p$ is the pressure, $\rho$ is the density, $\phi_{m o m}$ is the viscous dissipation, $q^{e}$ is the inlet heat flux from the surrounding, and $\dot{Q}$ is the heat source inside the body. Here we focus on the material body and leave out the heat transfer forms, for the conduction and convection can be included in $q^{e}$ and radiation can be included in $\dot{Q}$.

Since,

$$
\begin{gathered}
d U=c_{V}(T) d T+\left(T\left(\frac{\partial p}{\partial T}\right)_{v}-p\right) d v \\
\frac{d q^{e}}{d t}=-\nabla \cdot \boldsymbol{q}
\end{gathered}
$$

Eq. (1) can be written as,

$$
\rho c_{V}(T) \frac{d T}{d t}=-T\left(\frac{\partial p}{\partial T}\right)_{v} \nabla \cdot \boldsymbol{v}-\nabla \cdot \boldsymbol{q}+\phi_{m o m}+\dot{Q}
$$

Multiplying both sides of equation Eq. (4) by temperature $T$ givens an equation which can be viewed as the conservation equation of the entransy in the heat transfer process,

$$
\rho c_{V}(T) T \frac{d T}{d t}=-T^{2}\left(\frac{\partial p}{\partial T}\right)_{v} \operatorname{div} \boldsymbol{v}-\nabla \cdot(\boldsymbol{q} T)+\boldsymbol{q} \cdot \nabla T+\phi_{m o m} T+\dot{Q} T
$$

According to the definition of entransy, we have,

$$
g=\frac{1}{2} u T
$$

Thus, Eq. (5) becomes to,

$$
\frac{d g}{d t}=-\dot{g}_{w}-\nabla \cdot(\boldsymbol{q} T)-\phi_{h}+\dot{g}_{\tau}+\dot{g}_{h}
$$

where the left side of the equation is the rate of change of entransy inside the material body with time, $q T$ is the entransy 
flow density, $\dot{g}_{w}$ is the entransy change owing to volume variation, $\dot{g}_{\tau}$ is the entransy change owing to viscous dissipation, $\dot{g}_{h}$ is the entransy change owing to heat source and $\phi_{h}$ is the entransy dissipation rate per unit body defined in heat transfer process for incompressible and inviscid fluid, expressed as,

$$
\phi_{h}=-\boldsymbol{q} \cdot \nabla T
$$

Eq. (7) is the entransy balance equation for heat conduction and convection process of compressible viscous fluid.

Now consider a steady heat transfer process,

$$
\rho c_{V}(T) \frac{\partial T}{\partial t}=0
$$

Thus the entransy balance equation is,

$$
-\boldsymbol{q} \cdot \nabla T+\rho c_{V}(T) T \boldsymbol{v} \cdot \nabla T-\phi_{m o m} T+T^{2}\left(\frac{\partial p}{\partial T}\right)_{v} \nabla \cdot \boldsymbol{v}=-\nabla \cdot(\boldsymbol{q} T)+\dot{Q} T
$$

Integration of Eq. (10) over the entire heat transfer domain gives,

$$
\iiint_{V_{*}}\left[-\boldsymbol{q} \cdot \nabla T+\rho c_{V}(T) T \boldsymbol{v} \cdot \nabla T-\phi_{m o m} T+T^{2}\left(\frac{\partial p}{\partial T}\right)_{v} \operatorname{div} \boldsymbol{v}\right] d V=\iiint_{V_{*}}-\nabla \cdot(\boldsymbol{q} T) d V+\iiint_{V_{*}} \dot{Q} T d V
$$

Transforming the volume integral to a surface integral on the domain boundary by Gauss's Law gives the modified entransy dissipation rate in the entire heat conduction domain as,

$$
\begin{array}{r}
G_{m}=\iiint_{V_{*}}\left[-\boldsymbol{q} \cdot \nabla T+\rho c_{V}(T) T \boldsymbol{v} \cdot \nabla T-\phi_{\text {mom }} T+T^{2}\left(\frac{\partial p}{\partial T}\right)_{v} \operatorname{div} \boldsymbol{v}\right] d V \\
=\iint_{\Sigma_{*}} \boldsymbol{q}_{\text {in }} T_{\text {in }} d \Sigma-\iint_{\Sigma_{*}} \boldsymbol{q}_{\text {out }} T_{\text {out }} d \Sigma+\iiint_{V_{*}} \dot{Q} T d V
\end{array}
$$

Where subscript in, out represent the inflow and outflow through the boundaries and the corresponding temperature.

Total effective heat transfer inside the body can be expressed as,

$$
\dot{Q}_{t}=\iint_{\Sigma_{*}} \boldsymbol{q}_{\text {in }} d \Sigma+\iiint_{V_{*}} \dot{Q} d V=\iint_{\Sigma_{*}} \boldsymbol{q}_{\text {out }} d \Sigma
$$

Multi-dimensional heat transfer process needs a heat flux-weighted average temperature difference, defined as the ratio of the total entransy change rate and total heat transfer rate, 


$$
\Delta T=\frac{G_{m}}{\dot{Q}_{t}}=\iint_{\Sigma_{*}} \frac{\boldsymbol{q}_{\text {in }}}{\dot{Q}_{t}} T_{\text {in }} d \Sigma-\iint_{\Sigma_{*}} \frac{\boldsymbol{q}_{\text {out }}}{\dot{Q}_{t}} T_{\text {out }} d \Sigma+\iiint_{V_{*}} \frac{\dot{Q}}{\dot{Q}_{t}} T d V
$$

The divergence theorem is then used to derive the minimum entransy dissipation principle for steady-state heat transfer optimization at a given heat transfer rate, which can be expressed as,

$$
\dot{Q}_{t} \delta(\Delta T)=\delta \iiint_{V_{*}} g_{m} d V=0
$$

where $\delta$ is the variation operator.

When the boundary heat transfer rate is given, minimization of the entransy dissipation leads to minimization of the temperature difference. Conversely, for heat conduction at a given temperature difference, the maximum entransy dissipation principle can be written as,

$$
\Delta T \delta\left(\dot{Q}_{t}\right)=\delta \iiint_{V_{*}} g_{m} d V=0
$$

Eqs. (15) and (16) show that the entransy dissipation extreme leads to the best heat conduction performance with the maximum effective thermal conductance, i.e. the maximum heat flux for prescribed boundary temperature difference, or the minimum temperature difference for a given boundary heat flux or a prescribed internal heat source.

2.2 Field synergy equation for compressible viscous fluid laminar convection heat transfer

Heat transfer is a typical irreversible process, and in order to quantify the irreversibility, the concept of entransy is introduced. From the former part we concluded that during a heat transfer process of compressible viscous fluid, extreme entransy change rate corresponds to best heat transfer performance. The evaluation criterion for heat transfer performance can be written as,

$$
G_{m}=\iiint_{V_{*}} g_{m} d V=\iiint_{V_{*}}\left[-\boldsymbol{q} \cdot \nabla T+T \rho \boldsymbol{v} \cdot \nabla\left(c_{p} T\right)-T \phi_{m o m}+T^{2}\left(\frac{\partial p}{\partial T}\right)_{v} \operatorname{div} \boldsymbol{v}\right] d V
$$

The mechanical work maintaining the fluid flow equals to the integral of the viscous dissipation function over the 
whole domain, which need to remain constant,

$$
\iiint_{V_{*}} \phi_{m o m} d V=\text { const }
$$

where the viscous dissipation function of Newtonian fluid is,

$$
\begin{gathered}
\phi_{\text {mom }}=\mu(T)\left[2\left(\frac{\partial v_{x}}{\partial x}\right)^{2}+2\left(\frac{\partial v_{y}}{\partial y}\right)^{2}+2\left(\frac{\partial v_{z}}{\partial z}\right)^{2}+\left(\frac{\partial v_{x}}{\partial x}+\frac{\partial v_{y}}{\partial y}\right)^{2}+\left(\frac{\partial v_{z}}{\partial y}+\frac{\partial v_{y}}{\partial z}\right)^{2}\right. \\
\left.+\left(\frac{\partial v_{z}}{\partial x}+\frac{\partial v_{x}}{\partial z}\right)^{2}-\frac{2}{3}(\operatorname{div} \boldsymbol{v})^{2}\right]
\end{gathered}
$$

In addition, the convective heat transfer must satisfy the following equations,

The continuity equation:

$$
\operatorname{div}(\rho \boldsymbol{v})=0
$$

The energy conservation equation:

$$
\rho \boldsymbol{v} \cdot \nabla\left(c_{p} T\right)=-p \operatorname{div} v+\operatorname{div}(k \nabla T)+\phi_{m o m}
$$

Also, equation of state is needed to make sure the equations are close. Assume the equation of state (EOS) is,

$$
p(\rho, T)=0
$$

To get the optimal flow field, a Lagrange function which includes the objective and constraint functions is established as flows,

$$
\begin{aligned}
\Pi=\iiint\left[\left[k(\nabla T)^{2}\right.\right. & \left.+T \rho \boldsymbol{v} \cdot \operatorname{grad}\left(c_{p} T\right)-T \phi_{m o m}+T^{2}\left(\frac{\partial p}{\partial T}\right)_{v} \operatorname{div} \boldsymbol{v}\right]+C_{0} \Phi_{m o m} \\
& \left.+A\left(-p \operatorname{div} \boldsymbol{v}+\operatorname{div}(k \operatorname{grad} T)+\phi_{m o m}-\rho \boldsymbol{v} \cdot \operatorname{grad}\left(c_{p} T\right)\right)+B \operatorname{div}(\rho \boldsymbol{v})+D p(\rho, T)\right] \mathrm{d} V
\end{aligned}
$$

where $C_{0}, A, B, D$ are Lagrange multipliers. $C_{0}$ is required to be constant, while the other multipliers are functions of $v, T$, $\rho$ and the position $\boldsymbol{x}$.

The variation of $\Pi$ with respect to $v$,

$$
\begin{gathered}
\left(C_{0}+A-T\right)\left[-2\left(\mu^{\prime}(T)-\frac{2}{3} \mu(T)\right) \operatorname{grad} \operatorname{div} v-2 \operatorname{div} v \cdot \operatorname{grad}\left(\mu^{\prime}(T)-\frac{2}{3} \mu(T)\right)+2 \mu(T) \operatorname{grad} \operatorname{div} v\right. \\
+2 \operatorname{div} v \operatorname{grad} \mu(T)]-\rho(A-T) \operatorname{grad}\left(c_{p} T\right)-\rho \operatorname{grad} B+\operatorname{grad}(p A-p T)=0
\end{gathered}
$$


The variation of $\Pi$ with respect to $T$,

$$
\begin{gathered}
\frac{\partial p}{\partial T} D+T \rho \boldsymbol{v} \cdot \operatorname{grad} c_{p}-\phi_{m o m}+p \operatorname{div} \boldsymbol{v}-2 \operatorname{div}(k \operatorname{grad} T)+\rho \boldsymbol{v} c_{p} \cdot \operatorname{grad} A+\operatorname{div}(k \operatorname{grad} A)+\rho \boldsymbol{v} \\
\cdot \operatorname{grad}(A-T) \frac{\partial c_{p}(T)}{\partial T}+\left[(\operatorname{grad} T)^{2}-\operatorname{grad} T \cdot \operatorname{grad} A\right] \frac{\partial k(T)}{\partial T} \\
+\left(C_{0}+A-T\right)\left\{(\operatorname{div} \boldsymbol{v})^{2} \frac{\partial \mu^{\prime}(T)}{\partial T}+\frac{\phi_{m o m}}{\mu(T)} \frac{\partial \mu(T)}{\partial T}\right\}+\left[(T-A) \boldsymbol{v} \cdot \operatorname{grad}\left(c_{p} T\right)-\boldsymbol{v} \cdot \operatorname{grad} B+\frac{\partial p}{\partial \rho} D\right] \frac{\partial \rho(T)}{\partial T}=0
\end{gathered}
$$

The variation of $\Pi$ with respect to $\rho$,

$$
(T-A) \boldsymbol{v} \cdot \operatorname{grad} T-\boldsymbol{v} \cdot \operatorname{gradB}-\frac{\partial p}{\partial \rho} D=0
$$

From Eq. (26) we can get,

$$
D=\frac{(T-A) v \cdot \operatorname{grad}\left(c_{p} T\right)-v \cdot \operatorname{grad} B}{\frac{\partial p}{\partial \rho}}
$$

Comparing Eq. (24) with the momentum equation of variable physical properties,

$$
\begin{aligned}
\frac{1}{2\left(C_{0}+A-T\right)}(A-T)\left[\operatorname{grad}\left(c_{p} T\right)+p \operatorname{grad}\left(\frac{1}{\rho}\right)\right]-\frac{1}{2\left(C_{0}+A-T\right)} \operatorname{grad}\left[\frac{p(A-T)}{\rho}-B\right] \\
=\boldsymbol{F}-\frac{1}{\rho} \operatorname{grad} p-\boldsymbol{v} \operatorname{div} \boldsymbol{v}
\end{aligned}
$$

Thus,

$$
\operatorname{grad} B=\operatorname{grad} \frac{p(A-T)}{\rho}-\frac{2\left(C_{0}+A-T\right)}{\rho} \operatorname{grad} p
$$

And the additional force is:

$$
\boldsymbol{F}=\frac{1}{2\left(C_{0}+A-T\right)}(A-T)\left[\operatorname{grad}\left(c_{p} T\right)+p \operatorname{grad}\left(\frac{1}{\rho}\right)\right]+\boldsymbol{v} \operatorname{div} \boldsymbol{v}
$$

Therefore, the governing equation for the optimal velocity field could be further reduced to:

$$
\begin{gathered}
\rho \boldsymbol{v} \operatorname{div} \boldsymbol{v}=-\operatorname{grad} p \\
+\left[\mu^{\prime}(T)+\frac{1}{3} \mu(T)\right] \operatorname{grad} \operatorname{div} \boldsymbol{v}+\mu(T) \Delta \boldsymbol{v}+\operatorname{div} \boldsymbol{v} \cdot \operatorname{grad}\left[\mu^{\prime}(T)-\frac{2}{3} \mu(T)\right]+2 \operatorname{div} \boldsymbol{v} \operatorname{grad} \mu(T) \\
+\rho\left\{\frac{1}{2\left(C_{0}+A-T\right)}(A-T)\left[\operatorname{grad}\left(c_{p} T\right)+p \operatorname{grad}\left(\frac{1}{\rho}\right)\right]+\boldsymbol{v} \operatorname{div} \boldsymbol{v}\right\}
\end{gathered}
$$

And the parameter $A$ in Eq. (31) should satisfy flowing equation:

$$
\begin{gathered}
\frac{\partial p}{\partial T} D+T \rho \boldsymbol{v} \cdot \operatorname{grad} c_{p}-\phi_{m o m}+p \operatorname{div} \boldsymbol{v}-2 \operatorname{div}(k \operatorname{grad} T)+\rho \boldsymbol{v} c_{p} \cdot \operatorname{grad} A+\operatorname{div}(k \operatorname{grad} A)+\rho \boldsymbol{v} \\
\cdot \operatorname{grad}(A-T) \frac{\partial c_{p}(T)}{\partial T}+\left[(\operatorname{grad} T)^{2}-\operatorname{grad} T \cdot \operatorname{grad} A\right] \frac{\partial k(T)}{\partial T}
\end{gathered}
$$




$$
+\left(C_{0}+A-T\right)\left\{(\operatorname{div} v)^{2} \frac{\partial \mu^{\prime}(T)}{\partial T}+\frac{\phi_{m o m}}{\mu(T)} \frac{\partial \mu(T)}{\partial T}\right\}=0
$$

The definition of parameter D and B can be found in Eq. (27) and (29).

The velocity field satisfying Eq. (31) will lead to the best performance of heat transfer. That is to say, any changes of the velocity field could only weaken the heat transfer when the viscous dissipation maintains constant. In order to be distinguished from the current field synergy equation developed by Chen [16], the Eq. (31) is defined as the modified field synergy equation (MFSE) for convective heat transfer. The additional force $\boldsymbol{F}$ is named as the synergy force which is related to the velocity field and the temperature gradient field. It is a special force that drives the fluid flow in synergy with the heat transfer, that is, promotes the fluid to flow along the direction of the temperature gradient. It is a virtual additional force that makes the modified field synergy equation not the ordinary momentum equation. Similar to but not the same as the field synergy equation without volume variation, the space distribution of the synergy force is not only dependent on the characteristics of heat transport such as the distribution of temperature $T$, anti-temperature $A$, but also the characteristics of flow behavior, i.e. velocity $v$ and density $\rho$. The potential effect of synergy force is to drive fluid to flow along the heat flux or in the reverse direction. However, because of the constraint of the actual boundary, the optimum flowing pattern governed by the field synergy equation is generally more complicated. Another thing that needs to be emphasis is the additional force $\mathrm{F}$ does not exist in nature. Therefore, the optimum velocity field indicates the basic characteristics of the flow pattern that benefits the heat transfer mostly. If an enhancement element could create a velocity field similar to the optimum velocity field, it will have a very satisfactory performance in heat transfer and flow resistance. The most direct application of the synergy equation is to offer guidance for selecting and designing an appropriate enhancement technique.

It has to be emphasized that an equation of state should be carefully selected based on the working fluid. Then the equation of state needs to be solved with the governing equations listed before to get the optimized velocity field and temperature distribution. 
The differences among the mathematical model without optimization, optimized using FSE, and optimized using MFSE are listed in part 4.2.3.

\section{Numerical Model Development for Cavity Air Flow Using MFSE as the Optimization Method}

\subsection{Governing Equations for Compressible Cavity Air Flow}

The laminar convection heat transfer enhancement in 2D cavity is a classical problem with wide engineering applications. In this part, air is chosen to be the working fluid for illustrating the former derivation. Its motion is thus described by the Navier-Stokes equations completed by an equation of state. Although the compressibility of air is not that significant, the model, which is currently admitted, is still considered a compressible fluid with variable physical properties. This is because the simplicity of the problem and the convenience of the comparison. The equation of state for air is,

$$
p=\rho r T
$$

where,

$$
r=287\left[\mathrm{~m}^{2} \cdot \mathrm{s}^{-1} \cdot \mathrm{K}^{-1}\right]
$$

Because of the introduction of the equation of state, the governing equations developed in section 2.2 become to:

Continuity equation:

$$
\nabla \cdot(\rho \boldsymbol{v})=0
$$

Momentum equation:

$$
\begin{gathered}
\rho \boldsymbol{v} \operatorname{div} \boldsymbol{v}=-\operatorname{grad} p+\frac{1}{3} \mu(T) \operatorname{grad} \operatorname{div} \boldsymbol{v}+\mu(T) \Delta \boldsymbol{v} \\
+\rho\left\{\frac{1}{2\left(C_{0}+A-T\right)}(A-T)\left[\operatorname{grad}\left(c_{p} T\right)+p \operatorname{grad}\left(\frac{1}{\rho}\right)\right]+\boldsymbol{v} \operatorname{div} \boldsymbol{v}\right\}
\end{gathered}
$$

Energy equation: 


$$
\rho \boldsymbol{v} c_{p} \cdot \operatorname{div} T=-p \operatorname{div} \boldsymbol{v}+k \operatorname{div}(\operatorname{grad} T)+\phi_{m o m}
$$

Governing equation of parameter $A$ :

$$
\begin{aligned}
&-\rho \boldsymbol{v} \cdot \operatorname{grad} A=\frac{k}{c_{p}} \operatorname{div}(\operatorname{grad} A)-\frac{1}{c_{p}}\left\{\frac{\partial p}{\partial T} D-\phi_{\text {mom }}+p \operatorname{div} \boldsymbol{v}-2 \operatorname{div}(k \operatorname{grad} T)+\rho \boldsymbol{v}\right. \\
& \cdot \operatorname{grad}(A-T) \frac{\partial c_{p}(T)}{\partial T}+\left[(\operatorname{grad} T)^{2}-\operatorname{grad} T \cdot \operatorname{grad} A\right] \frac{\partial k(T)}{\partial T}+\left(C_{0}+A\right. \\
&\left.-T) \frac{\phi_{\text {mom }}}{\mu(T)} \frac{\partial \mu(T)}{\partial T}\right\}
\end{aligned}
$$

The definitions of parameter $D$ and $\operatorname{grad} B$ are shown in Eq. (27) and (29). $C_{0}$ is a parameter corresponding to viscous dissipation, and it's assumed to be constant during the simulation.

Altogether, Eqs (33) - (37) are the governing equations of the current problem. This optimization method is called the MFSE method in the later part.

\subsection{Numerical Process}

A cavity with $20 \mathrm{~mm}$ in length is selected to verify the model presented above. Both the flow and temperature are assumed to be fully developed. The upper and lower walls are isothermal, the wall temperature of left side is $300 \mathrm{~K}$ and of the right side is $305 \mathrm{~K}$. The thicknesses of the walls are neglected.

In order to get an accurate simulation result without useless time cost, grids are carefully selected. It is noticed that the calculation could be easily diverge because of the source items. It should be emphasized that the vortexes caused by these source items are not only near the heating and cooling boundary, but also in the interior. Mesh encryptions near the boundary would not be enough. An overall refined mesh is needed. In the study, effects of the grid number on the simulation accuracy are examined. The maximum deviations of the predicted local Nusselt number are less than 5\% among the computations on the grids of $188 \times 188,212 \times 212,228 \times 228,272 \times 272,364 \times 364$. Therefore, the grid system of $228 \times 228$ points seems to be sufficient.

The commercial software FLUENT 14.0 is employed for all the simulations. The additional source terms of the 
laminar field synergy equations (developed in [16]) and the modified field synergy equations (Eq. (33) - (37)) are added by the user-defined function (UDF).The cavity is modeled as a two-dimensional body according to Fig. 1. The laminar model is selected and the pressure and velocity are coupled by the SIMPLE algorithm. The diffusion and convection terms are discretized using a second order upwind scheme. A converged solution is obtained using the former mentioned grid, with root mean square residuals less than $10^{-7}$ for all the transport variables and independent of the iteration numbers, and domain imbalances of mass, momentum and energy conservation less than $10^{-7}$.

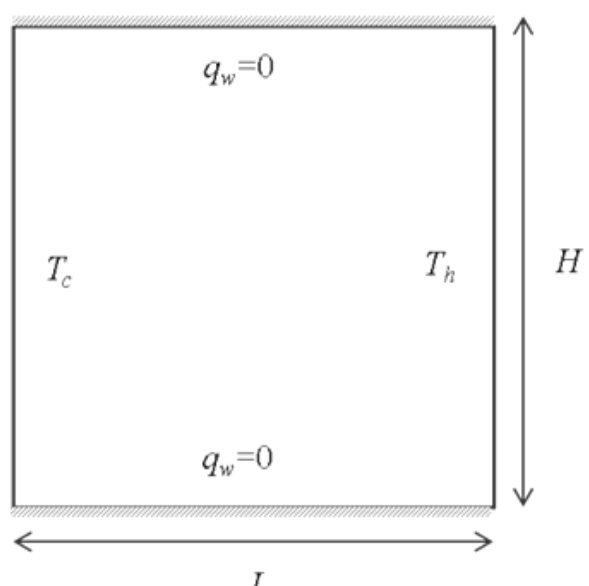

$L$

Fig. 1 The physical model of convectional heat transfer in a square cavity

\section{Numerical results and discussion}

\subsection{Results Comparison for Basic Case and LFSE-Optimized Results}

For the results before optimization, that is, the flow and heat transfer performance obtained by solving the N-S equation, the velocity vectors and the temperature gradients are shown in Fig 2. Because of the change of density in this temperature range, a weak natural convection occurs, leading to a vortex inside the cavity. The heat transfer rate is $0.137 \mathrm{~W}$ in the computational domain. The optimized velocity and temperature fields of the computation domain using the laminar field synergy equations for $C_{0}=-0.01$ are shown in Fig 3 . Compared to the original cases, the computational 
domain is filled with a much larger eddy, and the heat transfer rate increases to $0.213 \mathrm{~W}$.

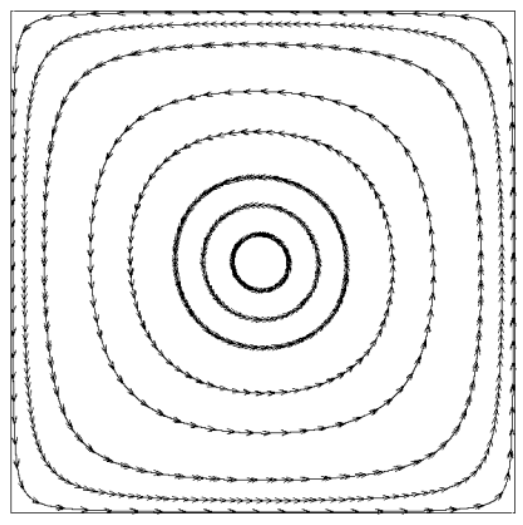

(a) streamline

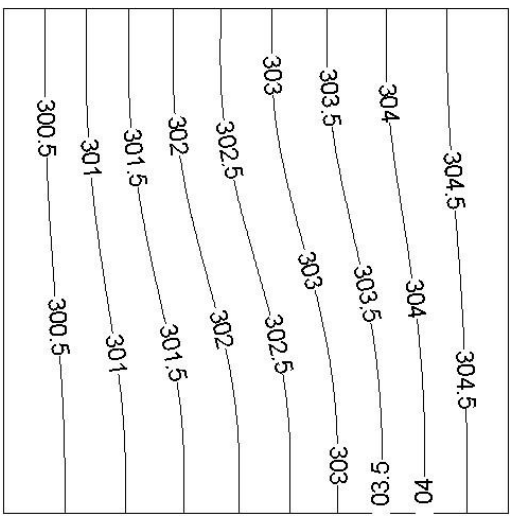

(b) temperature field $(\mathrm{K})$

Fig. 2 Streamline (left) and temperature field (right) of air in cavity $(20 \mathrm{~mm} \times 20 \mathrm{~mm})$ with no optimization.(a) streamline;

(b) temperature field

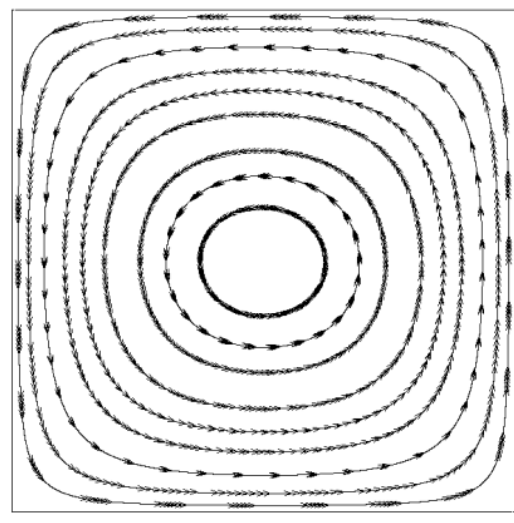

(a) optimal streamline

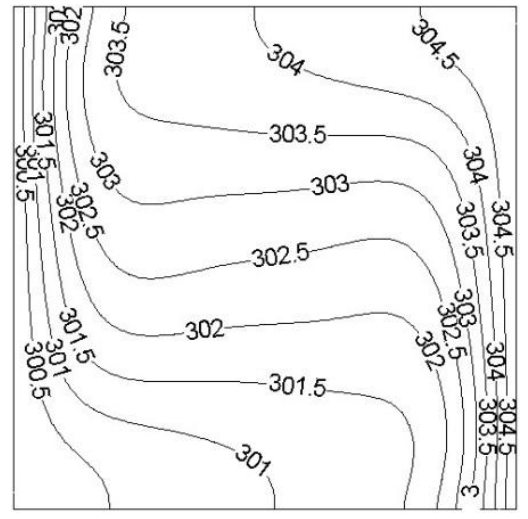

(b) optimal temperature field (K)

Fig. 3 Streamline (left) and temperature field (right) of air in cavity $(20 \mathrm{~mm} \times 20 \mathrm{~mm})$ using FSE (present) under $C_{0}$ $=-0.01$.(a) optimal streamline; (b) optimal temperature field (K)

\subsection{MFSE-Optimized Results}

Recall the momentum equation derived in the former part (eq. (31)), the additional body force is,

$$
\boldsymbol{F}=\rho \boldsymbol{v} \operatorname{div} \boldsymbol{v}+\rho \frac{1}{2\left(C_{0}+A-T\right)}(A-T) \operatorname{grad}\left(c_{p} T\right)
$$




$$
-\frac{1}{2\left(C_{0}+A-T\right)} \operatorname{grad}\left[T^{2} \frac{\partial p(\rho, T)}{\partial T}\right]-\frac{1}{2\left(C_{0}+A-T\right)} \operatorname{grad} A
$$

This body force is regarded as a source item in the simulation, and it can be divided into 4 items considering the meanings of each item. These items are the mass force (MF), the body force brought by internal energy change (BF-EC), the body force brought by density change (BF-DC) and the body force brought by the change of $A$ (BF-AC), respectively.

In order to understand the influences of each item in the source item of the momentum equation, the items are added one by one during the simulation. The stream lines and temperature distributions are shown in Fig 4.

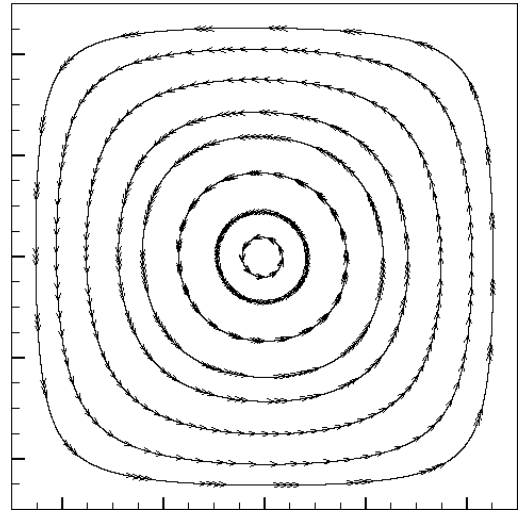

(a) the mass force

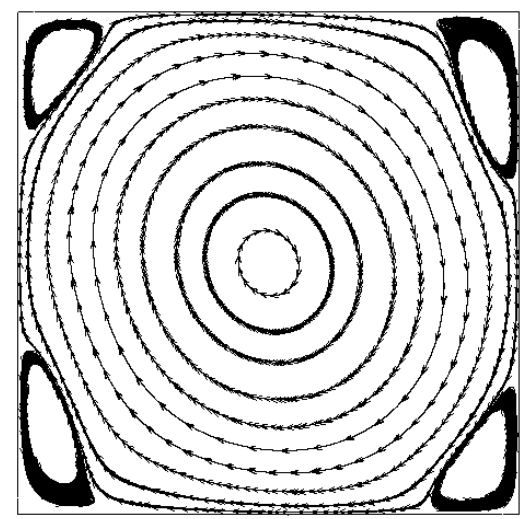

(c) the body force brought by density change

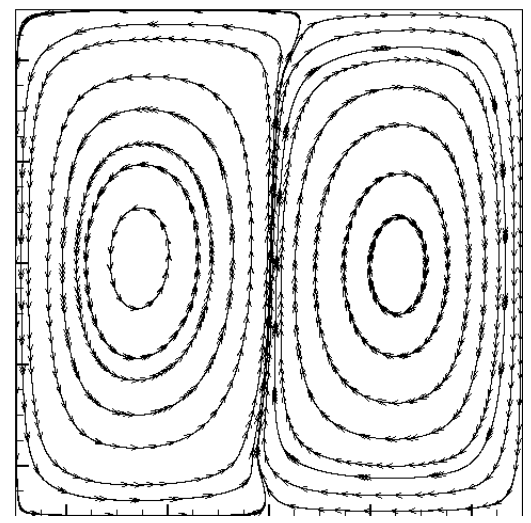

(b) the body force brought by internal energy change

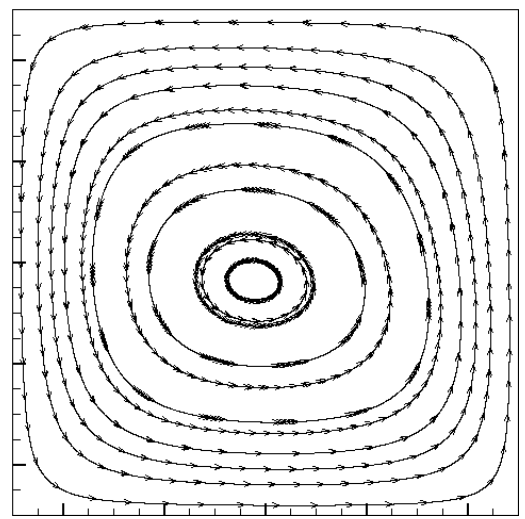

(d) the body force brought by $A$ change

Fig. 4 Optimized streamline of air in cavity $(20 \mathrm{~mm} \times 20 \mathrm{~mm})$ using modified FSE with different parts of the source item in the momentum equation added. (a)The mass force; (b) the body force brought by internal energy change; (c) the body force brought by density change; (d) the body force brought by $A$ change.

MF does not bring any changes to flow field and temperature distribution. BF-EC is considered as the effect of 
possible specific heat change. Different initial condition of flow and heat transfer can result in two different steady flow patterns: a flow field with two equirotal eddies or two eddies vary in size. The temperature field in this case is not significantly influenced because the slow clinal changes of the physical properties of air. The item BF-DC is the body force caused by the compressibility of fluid. The flow field is fully developed with a big vortex in the center and small symmetric vortexes in the corner. The temperature gradient near the boundary increases and the local heat transfer performance is significantly enhanced. BF-AC leads to a flat velocity field and the temperature field is twisted near the isothermal boundaries. In general, the additional body force leads to a much fierce convection. The hot and cold streams mix sufficiently and an enhanced heat transfer performance can be noticed.

\subsubsection{Effect of temperature difference}

A comparison between the temperature of the heating boundary of $305 \mathrm{~K}$ and $310 \mathrm{~K}$ under $C_{0}=-0.01$ can be noticed in

Fig. 5 and Fig. 6. The flow patterns and the temperature distributions are similar. Four small vortexes appear at each corner of the cavity. The increase of the temperature differences can lead to an increase of the size of the small vortexes. When the heating boundary temperature is $310 \mathrm{~K}$, the isothermal lines bend more severely comparing to that of $305 \mathrm{~K}$. The small vortexes enhance the heat transfer performance at the corner by destroy the boundary layer. The cold and hot stream mix significantly and the temperature field is fully developed.
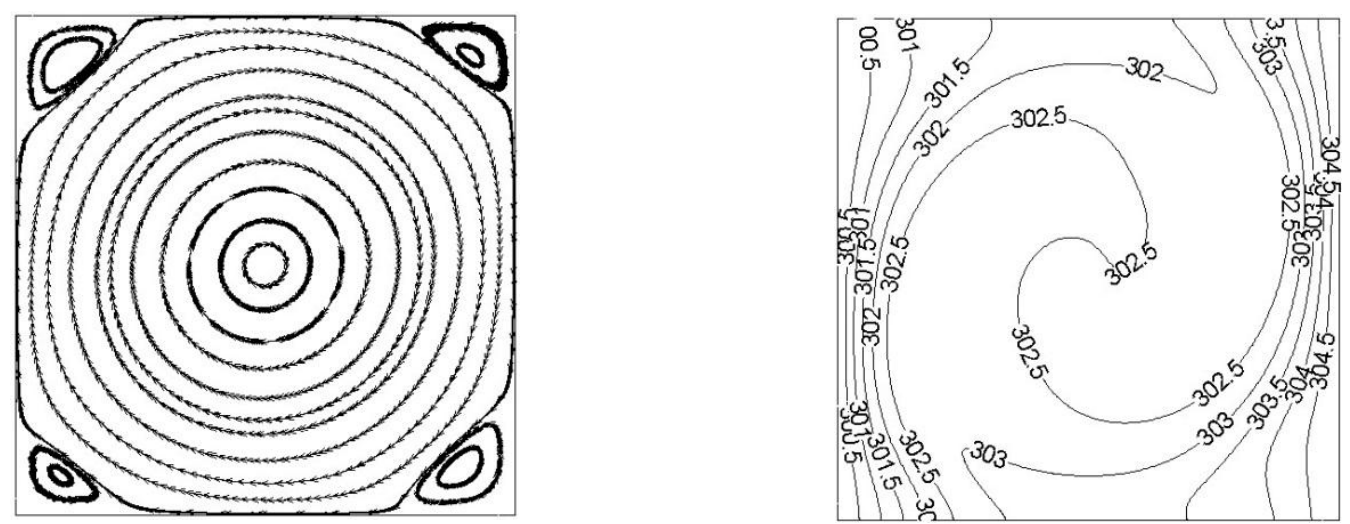
(a) optimal streamline

(b) optimal temperature field (K)

Fig. 5 Optimal streamline (left) and temperature field (right)of air in cavity $(20 \mathrm{~mm} \times 20 \mathrm{~mm})$ with $T_{h}=305 \mathrm{~K}$ using MFSE under $C_{0}=-0.01$.(a) optimal streamline; (b) optimal temperature field (K)

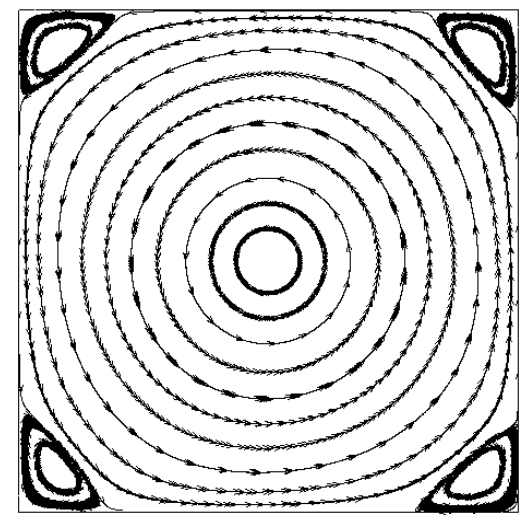

(a) optimal streamline

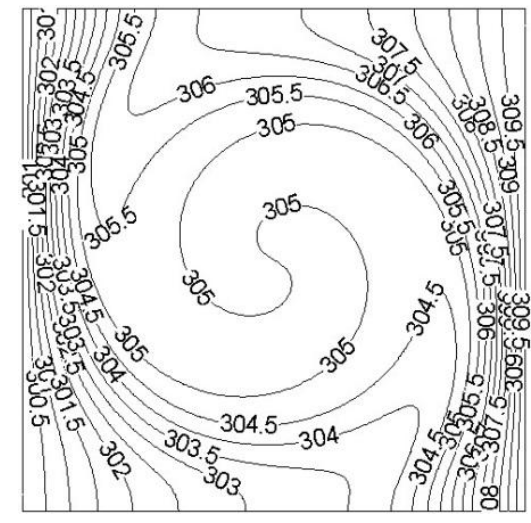

(b) optimal temperature field (K)

Fig. 6 Optimal streamline (left) and temperature field (right) of air in cavity $(20 \mathrm{~mm} \times 20 \mathrm{~mm})$ with $T_{h}=310 \mathrm{~K}$ using MFSE under $C_{0}=-0.01$. (a) optimal streamline; (b) optimal temperature field (K)

\subsubsection{Effect of viscous dissipation}

The parameter $C_{0}$ is set constant to make sure the viscous dissipation remain a specific value in the study. The increasing of the absolute value of $C_{0}$ means a larger viscous dissipation. Figure 7 shows the flow field and temperature distribution under $C_{0}=-0.1$. When $C_{0}$ increases, the number of the vortexes number decreases from four to two. The size of the vortexes in the corner is larger than that of $C_{0}=-0.01$. The vortex in the middle twists. The temperature field also twists because of the influence brought from the velocity field. The local $\mathrm{Nu}$ of the heating and cooling boundaries is plotted in Fig. 8. The local $N u$ reaches to its peak value because of the corner vortex. At the other two corners when no vortex exists, the isothermal lines are parallel to the streamline, which is not efficient in heat transfer. The valley value of the heat transfer rates appears in this area. 


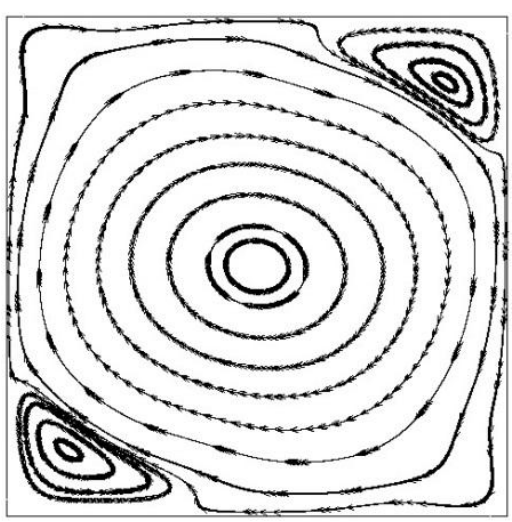

(a) optimal streamline

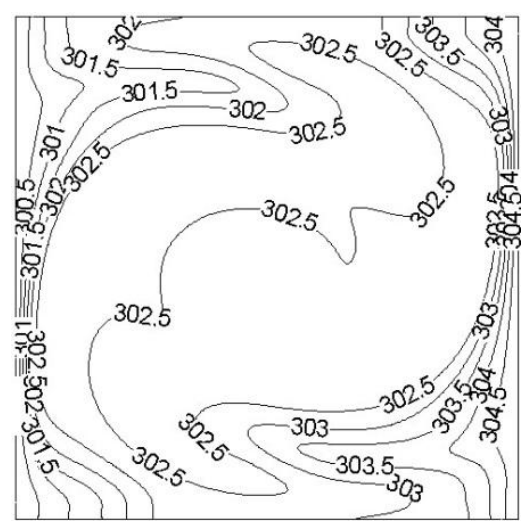

(b) optimal temperature field (K)

Fig. 7 Optimal velocity field (left) and temperature field (right) of air in cavity $(20 \mathrm{~mm} \times 20 \mathrm{~mm})$ with $T_{h}=305 \mathrm{~K}$ using MFSE under $C_{0}=-0.1$. (a) optimal streamline; (b) optimal temperature field (K)

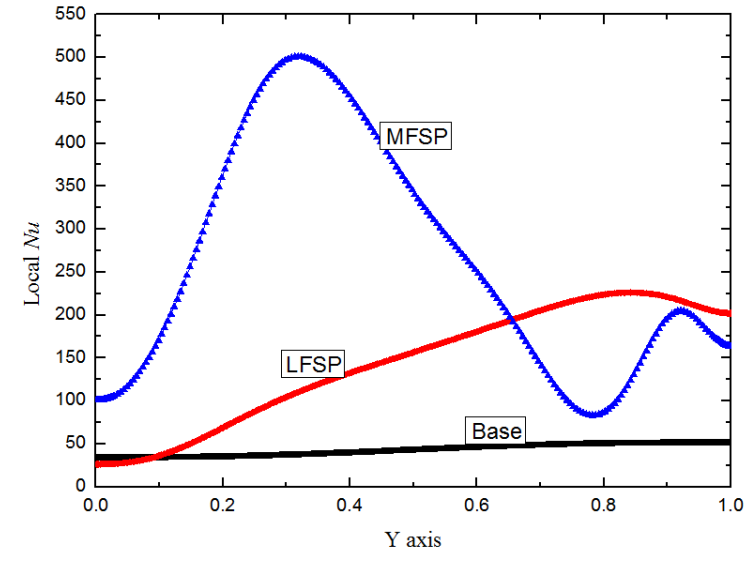

(a) the cooling boungdary

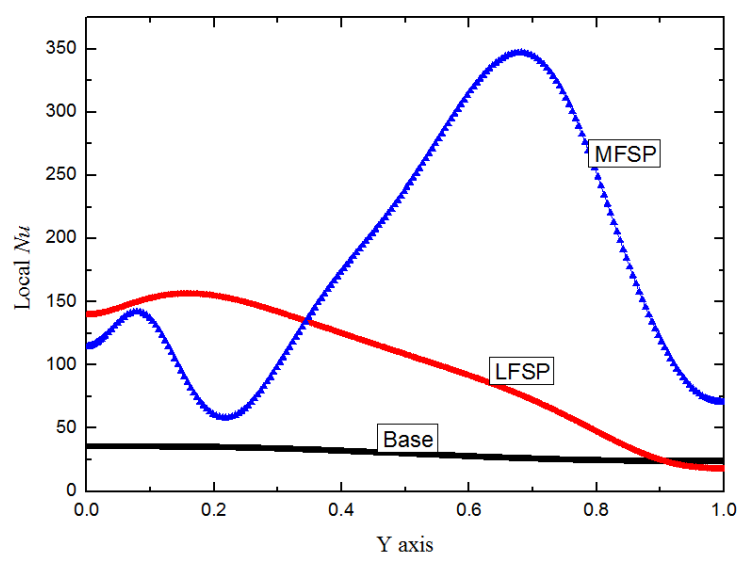

(b) the heating boundary

Fig. 8 The local $N u$ of the heating and cooling wall without optimization, optimized using LFSP and optimized using MFSP with $T_{h}=305 \mathrm{~K}$ under $C_{0}=-0.1$. (a) the cooling boungdary; (b) the heating boundary.

\subsubsection{Differences between the two optimization methods}

Table 1 shows a comparison between the original results, the optimized result using LFSE, and MFSE under different conditions. The total heat flux of the optimized results of the heating temperature equals to $305 \mathrm{~K}$ and $310 \mathrm{~K}$ using LFSE have increased by $55 \%$ and $57 \%$, respectively. The results of using MFSE have increased by $58 \%$ and $61 \%$, respectively. It is obvious that the equations developed in this study corresponding to the best heat-transfer performance when the 
density of fluid changes with temperature and pressure. When the viscous dissipation increases, the heat transfer performance increases significantly. And also, acute density change increases the difference between the two optimization equations.

Table 1 Comparison amongthe original result, optimized result using laminar FSE andMFSE with different boundary conditions under different $C_{0}$

\begin{tabular}{|c|c|c|c|c|}
\hline Heat transfer rate $(\mathrm{W})$ & $C_{0}$ & No Optimization & Laminar FSE & MFSE \\
\hline $\operatorname{Air}\left(T_{h}=305 \mathrm{~K}\right)$ & -0.01 & 0.137 & 0.213 & 0.219 \\
\hline $\operatorname{Air}\left(T_{h}=305 \mathrm{~K}\right)$ & -0.1 & 0.137 & 0.636 & 0.875 \\
\hline $\operatorname{Air}\left(T_{h}=310 \mathrm{~K}\right)$ & -0.01 & 0.292 & 0.458 & 0.471 \\
\hline
\end{tabular}

The differences among the model without optimization, model optimized using LFSE, and optimized MFSE are compared in Table 2. The biggest difference among these three models is the equations that are solved. The N-S equation is used to obtain the real flow pattern in the system while the laminar and modified field synergy equations is used to obtain an optimized flow field, which doesn't exist naturally in the current situation. The flow field is optimized because the constraint conditions have been added when the field synergy equation is derived.

Table 2 Differences amongthe model without optimization, model optimized using laminar FSE, and optimized MFSE (FSE for compressible fluid)

\begin{tabular}{lccc}
\hline & No Optimization & Laminar FSE & MFSE \\
\hline \multirow{2}{*}{ Assumptions } & -- & Laminar, incompressible, constant & Laminar, compressible, variable \\
& & properties & properties \\
\hline
\end{tabular}




\begin{tabular}{cccc}
$\begin{array}{c}\text { Momentum } \\
\text { Equation }\end{array}$ & N-S equation & Laminar field synergy equation & Modified field synergy equation \\
\hline $\begin{array}{c}\text { Equation of } \\
\text { state }\end{array}$ & -- & unnecessary & necessary \\
\hline Additional & -- & Governing equation of parameter $A$ & Modified governing equation of \\
equation & - & & parameter $A$ \\
\hline
\end{tabular}

\subsection{Further Discussions on Compressible Fluid Heat Transfer Optimization}

The derivation in this study used the definition of entransy for reference. When deducting the optimization criteria, we used the general energy conservation equation as a starting point to avoid the possible inaccuracy brought by the current definitions. Due to the influence of the temperature-dependent density change, the theoretical maximum value of heat transfer rate of the case discussed is increased, and this influence can be more obvious when the fluid is more compressible. But it should be emphasis that the numerical simulation is easy to diverge because of the additional source items. These items are larger when the compressibility of the working fluid is more significant, which may lead to the convergence problems. We separated the source items according to their physical meanings and added them one by one to avoid huge perturbations during the simulation. The convergence problem should be noticed when using this method for heat transfer optimization. Also, the whole derivation is based on the N-S equation, which means adjustment needs to be taken for turbulent equations.

The most important thing for system optimization is to define the optimized object and the constraint condition. In the present study, the entransy dissipation value is proposed as the optimized object, and the viscous dissipation value is set as the constraint condition. It is notable that other parameters could also be set as the optimized objects and the constraint condition. The specific parameters are chose in this study only because they are intuitive and easy to be quantified. 


\section{Conclusions}

Based on the concept of entransy, the field synergy equation of compressible viscous fluid with variable properties was developed together with the continuity and energy equation and the equation of state, and it was solved to predict the optimum heat transfer flow field of compressible viscous flows.

For compressible working fluid, change in temperature and pressure can significantly affect its density, which leads to volume change and results in volume work. In the study, a comparison between the results using the N-S equations, optimized results using existed laminar field synergy equation, and the modified field synergy equations of compressible condition with different temperature differences has been made. The result shows that the equations developed in this study corresponding to the best heat-transfer performance when the density of fluid changes with temperature and pressure.

Air flow in cavity is used as an example. It is found that the optimized flow field is the one with four small vortexes at the corner and a big one in the center, which significantly improved the heat transfer performance of the system. The results proved the availability of the modified field synergy equations and shown a possibility of optimizing the heat transfer performance under compressible condition. In practical engineering applications, the resulted optimal velocity fields can shed some predictive light on designing for heat exchange operations.

\section{Acknowledgement}

The support of National Science Foundation of China (No.51276001) and the Common Development Fund of Beijing are gratefully acknowledged.

\section{Nomenclature}


A

B

$C_{0}$

$c_{p}$

$c_{v}$

D

F

G

$g$

$k$

p

$Q$

$q$

$r$

$T$

$t$

$U$

$v_{x}, v_{y}, v_{z} \quad$ velocity component on $x, y, z$ direction, $\mathrm{m} \mathrm{s}^{-1}$

v velocity, $\mathrm{m} \mathrm{s}^{-1}$

\section{Greek symbols}

$\delta$

$\mu$

$\mu^{\prime}$

$\rho$

$\Phi$

dissipation rate, $\mathbf{J}$

\section{Subscripts}

c

cooling side

$e$

external source

$h$

heating side

heat

heat transfer process

in

inlet

modified

$m$

mom

momentum

outlet

total value

constant volume

volume change

viscous dissipation

\section{References}

[1] AE Bergles, Some perspectives on enhanced heat transfer - second generation heat transfer technology, J. Heat Transf. 
- Transactions of the ASME 1988, 110 (4B) 1082-1096.

[2] AE. Bergles, Heat transfer enhancement - the encouragement and accommodation of high heat fluxes. J. Heat Transf. - Transactions of the ASME 1997, 119 (1) 8-19.

[3] RL. Webb. Principles of enhanced heat transfer. New York: John Wiley\&Sons, 1994.

[4] A. Bejan, A study of entropy generation in fundamental convective heat transfer. Journal of Heat Transfer Transactions of the ASME 1979,101 (4) 718-725.

[5] A. Bejan, Entropy generation through heat and fluid flow. New York: JohnWiley \& Sons; 1982.

[6] M. Mohsenia, M. Bazargan, Entropy generation in turbulent mixed convection heat transfer to highly variable property pipe flow of supercritical fluids, Energ. Convers. Manage. 87 (2014) 552-558.

[7] Y. Haseli, Performance of irreversible heat engines at minimum entropy generation, Appl. Math. Model. 37 (2013) 9810-9817.

[8] H. Bispo, N Silva, R. Brito, J. Manzi, On the equivalence between the minimum entropy generation rate and the maximum conversion rate for a reactive system, Energ. Convers. Manage. 76 (2013) 26-31.

[9] D.P. Sekulic, The second law of energy transformation in a heat exchanger, J. Heat Transf. 112 (1990) $295-300$.

[10] J.E. Hesselgreaves, Rationalization of second law analysis of heat exchangers, Int. J. Heat Mass Transf. 43 (2000) 4189-4204.

[11] K. Ogiso, Duality of heat exchanger performance in balanced counter-flow systems, J. Heat Transf. 125 (2003) 530-532.

[12] U. Lucia, Entropy generation: Minimum inside and maximum outside, Physica A 396 (2014) 61-65.

[13] Z.Y. Guo, X.G. Cheng, Z.Z. Xia, Least dissipation in principle of heat transport potential capacity and its application in heat conduction optimization, Chinese Science Bulletin 48 (2003) 406-410.

[14] Z.Y. Guo, H.Y. Zhu, X.G. Liang, Entransy-A physical quantity describing heat transfer ability, Int. J. Heat Mass 
Transf. 50 (2007) 2545-2556.

[15] Q. Chen, J.X. Ren, J.A. Meng. Field synergy equation for turbulent heat transfer and its application, Int. J. Heat Mass Transf. 50 (2007) 5334-5339.

[16] X.W. Li, J.A. Meng, Z.Y. Guo. Turbulent flow and heat transfer in discrete double inclined ribs tube. Int. J. Heat Mass Transf. 52 (2009) 962-970.

[17] J. Wu, X.G. Liang. The application of entransy dissipation extremum principle in radiative heat transfer optimization. Science in China Series E - Technological Sciences 51 (2008) 1306-1314.

[18] L. Chen, X.R. Zhang, Experimental Analysis on a Novel Solar Collector System achieved by Supercritical CO2 Natural Convection, Energy Convers. Mgmt. 77 (2014) 173-182.

[19] L. Chen, Y.M. Chen, M.H. Sun, Y.L Zhang, X.R. Zhang, Concept Design and Formation of a Double Effect Lithium Bromide-Water Cooling System Powered by Supercritical CO2 Solar Collector, Energy Convers. Mgmt. 85 (2014) 313-322.

[20] L. Chen, B.L. Deng, X.R. Zhang, Experimental Investigation of CO2 thermosyphon Flow and Heat Transfer in the Supercritical Region, Int. J. Heat Mass Transf. 64 (2013) 202-211.

[21] C. Nonino, S. Del Giudice, S. Savino, Temperature dependent viscosity effects on laminar forced convection in the entrance region of straight ducts, Int. J. Heat Mass Transf. 49 (2006) 4469-4481.

[22] S. Del Giudice, C. Nonino, S. Savino, Effects of viscous dissipation and temperature dependent viscosity in thermally and simultaneously developing laminar flows in microchannels, Int. J. Heat Fluid Flow 28 (2007) 15-27.

[23] V. Kumar, P. Gupta, K.D.P. Nigam, Fluid flow and heat transfer in curved tubes with temperature-dependent properties, Int. Eng. Chem. Res. 46 (2007) 3226-3236.

[24] L. Chen, X.R. Zhang, J. Okajima, S. Maruyama, Thermal Relaxation and Critical Instability of Near-Critical Fluid Microchannel Flow, Phys. Rev. E 87(2013) 043016 
[25] Q. Chen, M. Wang, N. Pan, Z.Y. Guo, Optimization principles for convective heat transfer, Energy 34 (2009) 1199-1206.

[26] B. Zappoli, Near-critical fluid hydrodynamics, C. R. Mecanique 331 (2003) 713-726.

[27] L. Chen, B.L. Deng, B. Jiang, X.R. Zhang, Thermal and Hydrodynamic Characteristics of Supercritical CO2 Natural Circulation in Closed Loops, Nucl.Eng. Des. 257 (2013) 21-30.

[28] L. Chen, X.R. Zhang, H. Yamaguchi, Z.S. (Simon) Liu, Effect of heat transfer on the instabilities and transitions of supercritical CO2 flow in a natural circulation loop, Int. J. Heat Mass Transf. 53 (2010) 4101-4111. 